\title{
Improved Cooperativity of Spin-Labile Iron(III) Centers by Self-Assembly in Solution
}

\author{
Claudio Gandolfi, ${ }^{\dagger}$ Christian Moitzi, ${ }^{\ddagger}$ Peter Schurtenberger, ${ }^{\ddagger}$ Grace G. Morgan, ${ }^{\S}$ and \\ Martin Albrecht ${ }^{*, \dagger}$ \\ Department of Chemistry and Adolphe Merkle Institute, University of Fribourg, CH-1700 Fribourg, Switzerland, \\ and UCD School of Chemistry \& Chemical Biology, University College Dublin, Belfield, Dublin 4, Ireland
}

We report on the markedly increased spin crossover activity of materials when self-assembled in solution. Spin crossover is directly related to long-range elastic interactions, typically observed in the solid state, and the transition may be gradual over a large temperature range. ${ }^{1}$ Effects such as solvent cocrystallization often modify the transition in an unpredictable way. This is predominantly because spin transitions are crucially affected by intermolecular interactions beyond crystal packing effects, such as elastic strain. ${ }^{2}$ Such intermolecular interactions are the classical domain to be engineered by supramolecular chemistry. ${ }^{3}$ Specifically, the promotion of close metal-metal interactions may stimulate cooperative behavior, which is expected to result in more abrupt spin transitions. This concept has been implemented for engineering of spin crossover active materials in the solid state ${ }^{4}$ and recently also in soluble nanoparticles. ${ }^{5}$ Here, we have used a hydrophilic/lipophilic bias $^{6}$ for inducing a favorable arrangement of potentially spin-labile iron(III) centers. Supramolecular organization in solution leads, most remarkably, to a new type of magnetically active material for liquidphase processing.

The $\mathrm{Fe}\left(\mathrm{sal}_{2}\right.$ trien) complex $\mathbf{2} \mathbf{a}$ is known to undergo a gradual spin transition in the solid state. ${ }^{7}$ Ligand functionalization and introduction of a lipophilic side chain was accomplished by selective alkylation of 4-hydroxysalicylaldehyde. Condensation of the aldehyde 1 with triethylenetetramine and subsequent metalation with iron(III) nitrate, either in situ or after isolation of the hexadentate ligand precursor, afforded the alkyl-tailed complexes $\mathbf{2} \mathbf{b}-\mathbf{d}$ (Scheme 1). Metathesis of the noncoordinating anion was straightforward because of the different solubility properties of $\mathrm{KPF}_{6}$ and the alkyltailed iron(III) complexes and gave, after extraction, complexes 3. A single crystal structure determination of complex $\mathbf{3 c}$ unambiguously confirmed the connectivity pattern (Figure 1a). The bond lengths around the two crystallographically independent iron centers are diagnostic for high spin $\mathrm{Fe}^{\mathrm{III}}$ at $173 \mathrm{~K}^{1 \mathrm{c}}$ The mutual cis coordination of the phenolate moieties arranges the alkyl chains in the same direction and maximizes intermolecular van der Waals interactions. As a consequence, the crystal packing displays apolar and polar layers consisting of the lipophilic side chains, and the charged metal coordination site and the $\mathrm{PF}_{6}{ }^{-}$anions, respectively (Figure 1b).

Despite the layered crystal packing, complex $\mathbf{3 c}$ does not exhibit thermochromic behavior in the solid state. Magnetic measurements indicate the absence of thermally induced spin crossover in the $30-300 \mathrm{~K}$ range and antiferromagnetism or zero-field splitting below $30 \mathrm{~K}^{1 \mathrm{c}}$ Similar results have been obtained for complexes $\mathbf{2 b}-\mathbf{d}$. This suggests that alkyl functionalization in $\mathbf{2}$ generates spinstable $\mathrm{Fe}^{\mathrm{III}}$ centers. The spin stability of $\mathbf{2 d}$ in the solid state may

\footnotetext{
† Department of Chemistry, University of Fribourg.

* Adolphe Merkle Institute, University of Fribourg.
\& University College Dublin.
}

Scheme 1. Synthesis of Functionalized Fe(sal 2 trien) Complexes
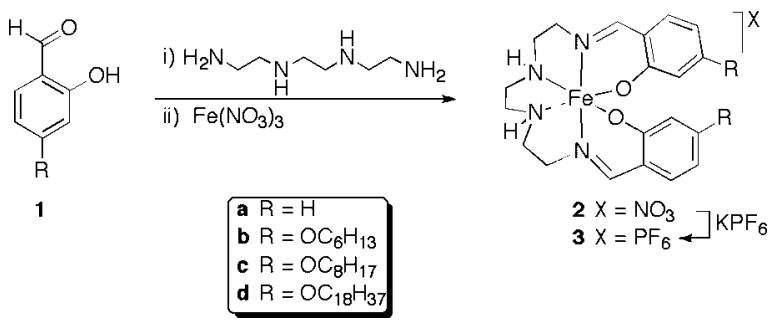

also originate from the absence of favorable metal-metal interactions, as powder diffraction showed this complex to be amorphous.

In contrast to the spin stability in the solid state, complex $\mathbf{2 d}$ undergoes spin crossover in solution. The spin transition is indicated by thermochromic behavior with a color change from red at room temperature to dark blue when cooled below $230 \mathrm{~K}\left(\mathrm{CH}_{2} \mathrm{Cl}_{2}\right)$. A similar color change was reported for $\mathbf{2 a}$ at $193 \mathrm{~K}^{7}$ The thermochromism was associated with a spin transition from high spin (HS; $S=5 / 2$ ) to low spin (LS; $S=1 / 2$ ). The new absorption band of $2 d$ in the LS state at $\lambda_{\max }=650 \mathrm{~nm}$ (inset Figure 2) reflects an energetically easier metal-to-ligand charge transfer, presumably due to a contraction of the $\mathrm{M}-\mathrm{L}$ bonds upon spin crossover.

Temperature-dependent monitoring of the extinction at $650 \mathrm{~nm}$ indicated a relatively abrupt transition around $230-240 \mathrm{~K}$ for $\mathbf{2 d}$ (Figure 2). Superimposition of the UV-vis spectra at different temperatures revealed an isosbestic point at $\lambda=558 \mathrm{~nm}(\epsilon=3700$ $\mathrm{M}^{-1} \mathrm{~cm}^{-1}$ ), thus providing evidence for an equilibrium between the HS and the LS states. Hence, the two spin states of $\mathbf{2 d}$ are distinctly
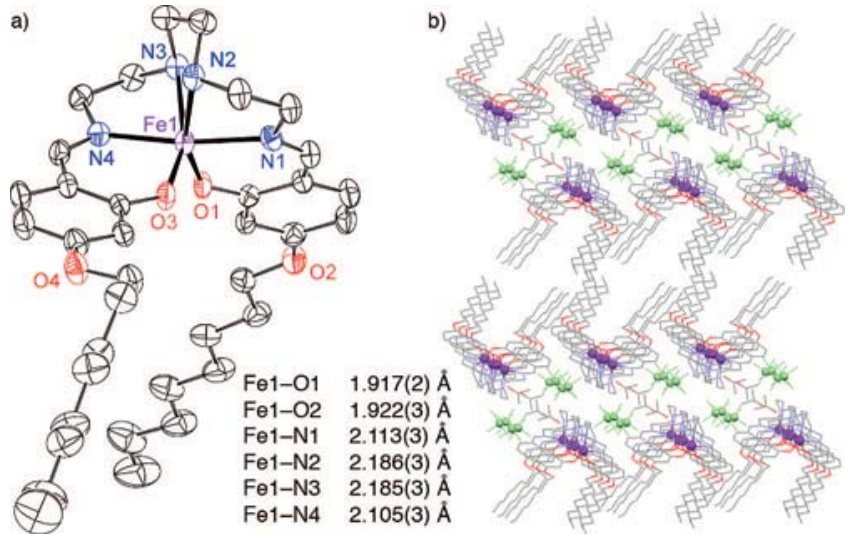

Figure 1. (a) ORTEP plot (50\% probability) and pertinent bond lengths of one of the two crystallographically independent cations of $3 \mathbf{c}$ at $173 \mathrm{~K}$ (hydrogen atoms omitted for clarity); (b) packing diagram of crystals of $\mathbf{3 c}$ illustrating the polar and apolar layers of $\mathbf{3 c}$ in the solid state (Fe, purple; $\mathrm{N}$, blue; $\mathrm{O}$, red; $\mathrm{PF}_{6}$, green). 


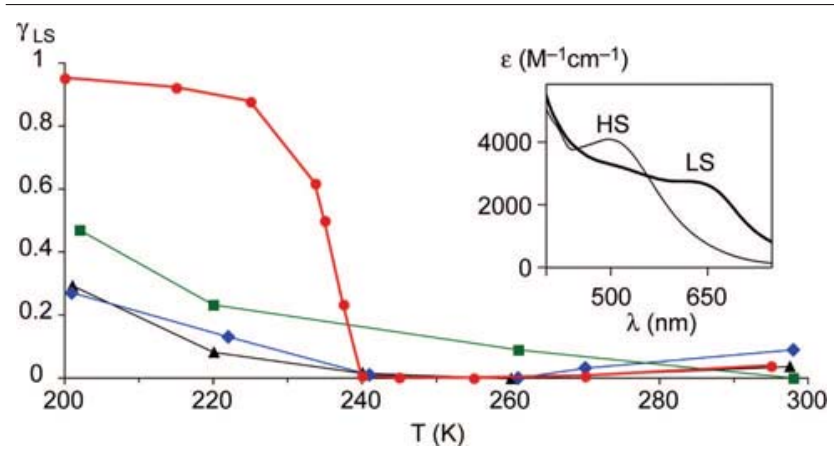

Figure 2. Fraction of LS complex $\left(\gamma_{\mathrm{LS}}\right)$ deduced from temperaturedependent $\mathrm{UV}-$ vis absorption at $650 \mathrm{~nm}$ for $\mathbf{2 a}(\mathbf{\square}), \mathbf{2 b}(\bullet), \mathbf{2 c}(\mathbf{\Delta}), \mathbf{2 d}$

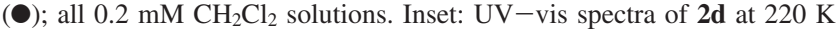
(LS, bold line) and $298 \mathrm{~K}$ (HS).

separated on the UV-vis time scale. No significant hysteresis has been observed upon repetitive heating-cooling cycles. Notably, the parent $\mathrm{Fe}$ ( $\mathrm{sal}_{2}$ trien) complex 2a, displays a gradual spin transition in solution over a $100 \mathrm{~K}$ temperature range. Similarly, no thermochromism was observed until $180 \mathrm{~K}$ for complexes $\mathbf{2 b}$ or $\mathbf{2 c}$ comprising shorter alkyl tails, suggesting that the alkyl chain length is crucial for promoting an abrupt spin transition.

Magnetic measurements confirmed the correlation between the color change and a spin crossover event. While the HS Fe ${ }^{\text {III }}$ signal could not be detected by EPR, the increasing isotropic resonance at $g=2.16\left(\mathrm{CH}_{2} \mathrm{Cl}_{2}\right)$ upon cooling is diagnostic for the population of a LS Fe ${ }^{\mathrm{III}}$ species. ${ }^{8}$ The high-spin nature of complex $\mathbf{2 d}$ at room temperature was evidenced by determining the magnetic susceptibility using Evans' method. ${ }^{9}$ At $298 \mathrm{~K}, \mu_{\text {eff }}=5.47 \pm 0.16 \mu_{\mathrm{B}}$ as expected for an $S=5 / 2$ configuration of spin crossover $\mathrm{Fe}^{\mathrm{III}}$. Unfortunately, the limited solubility of $\mathbf{2} \mathbf{d}$ in $\mathrm{CH}_{2} \mathrm{Cl}_{2}$ precluded ${ }^{1} \mathrm{H}$ NMR measurements at low temperature, and saturated solutions of $\mathbf{2 d}$ at $230 \mathrm{~K}$ were too dilute to induce an accurately defined paramagnetic shift of the solvent signal. ${ }^{10}$

The abrupt spin transition of $\mathbf{2 d}$ within $10 \mathrm{~K}$ suggests strongly cooperative behavior of the metal centers, which may be induced for example by self-organization of the molecules into supramolecular assemblies. Indeed, DLS measurements of solutions of $\mathbf{2 d}$ revealed the presence of particles with hydrodynamic radius $R_{\mathrm{H}} \approx$ $550 \mathrm{~nm}$ and a large polydispersity. Analyses by cryogenic scanning electron microscopy (SEM) showed two major types of spherical particles, characterized by an average diameter of ca. 1 and $3 \mu \mathrm{m}$, respectively (Figure 3). While these results corroborate the DLS measurements, the SEM plots further suggest the particles to be superstructures composed of rodlike assemblies with a diameter of about $30 \mathrm{~nm}$ (inset Figure 3). High intensity SAXS experiments using synchroton irradiation of $\mathbf{2 d}$ are consistent with elongated objects featuring a cross-section diameter of $42 \mathrm{~nm}$. Supercoils similar to DNA superstructures rather than simple micelle-type aggregates may account for the observed size of these nanorods (calculated length of $\mathbf{2 d}$ in a fully stretched conformation is $30 \AA$ ).

No such distinct structures were observed with complexes $\mathbf{2 b}$ and $2 \mathrm{c}$ comprising shorter alkyl tails. This suggests that the abrupt spin crossover, exclusively observed with $\mathbf{2 d}$, is correlated with the supramolecular assembly of this compound in solution to form discrete aggregates. Such a structural model may rationalize the intermolecular cooperativity required for the observed abrupt spin transition in solution.

In conclusion, our results demonstrate that supramolecular principles can be successfully applied to engineer metal-centered function, as illustrated by the enhanced cooperativity and higher transition temperature induced in an originally poorly cooperating spin crossover

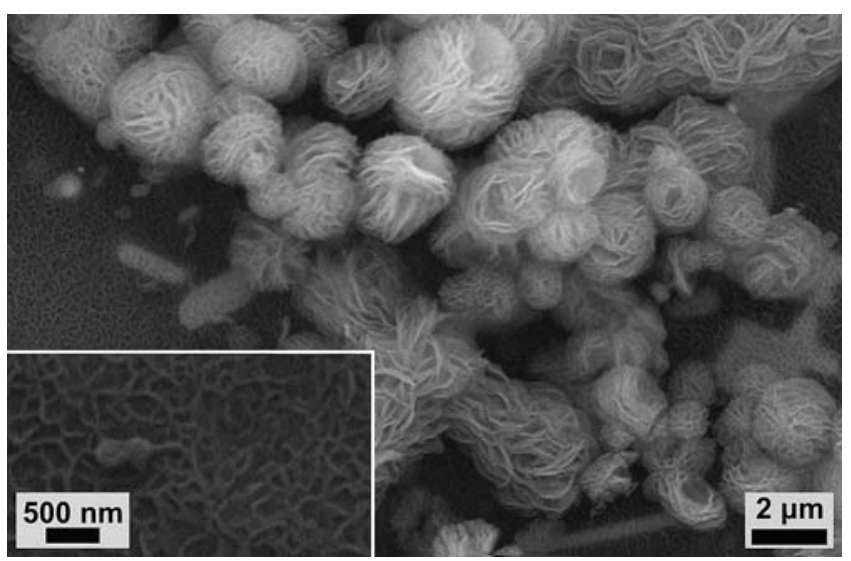

Figure 3. Cryo-SEM images of a representative section of self-assembled 2d; two types of spherical particles are distinguishable featuring diameters of ca. 1 and $3 \mu \mathrm{m}$, respectively. Inset: nanosize rods as subcomponents of spherical microparticles.

iron(III) complex by self-assembly. Control and improvement of such function in solution is achieved through careful chemical modulation. We are currently investigating the generality of this approach for fabricating magnetically active materials for solution processing, ideally operating near room temperature.

Acknowledgment. We thank Dr. M. M. Dadras (CSEM Neuchatel), Mr. V. K. Malik (Univ Fribourg), Dr. A. Menzel (PSI Villingen) for analytical measurements, Prof. H. Stoeckli-Evans (BENEFRI Crystallography Service, Univ Neuchâtel) for the crystal structure analysis, and Prof. C. W. Schläpfer for fruitful discussions. This work was financially supported by ERA-net Chemistry. M.A. gratefully acknowledges an Alfred Werner Assistant Professorship.

Supporting Information Available: Synthetic details and analytical data of complexes $\mathbf{2 b}-\mathbf{2 d}$, CIF for $\mathbf{3 c}$. This material is available free of charge via the Internet at http://pubs.acs.org.

\section{References}

(1) (a) Kahn, O.; Martinez, C. J. Science 1998, 279, 44. (b) Gütlich, P.; Garcia, Y.; Spiering, H. In Magnetism: Molecules to Materials IV; Miller, J. S., Drillon, M., Eds.; Wiley-VCH: Weinheim, Germany, 2002; p 271. (c) van Koningsbruggen, P. J.; Maeda, Y.; Oshio, H. Top. Curr. Chem. 2004, 233 , 259. (d) Spiering, H. Top Curr Chem 2004, 235, 171 (e) Letard, J.-F.; Guionneau, P.; Goux-Capes, L. Top. Curr. Chem. 2004, 235, 221.

(2) Hostettler, M.; Törnroos, K. W.; Chernyshov, D.; Vangdal, B.; Bürgi, H.B. Angew. Chem., Int. Ed. 2004, 43, 4589.

(3) Lehn, J.-M. Supramolecular Chemistry; Wiley-VCH: Weinheim, Germany, 1995.

(4) (a) Real, J. A.; Andres, E.; Munoz, M. C.; Julve, M.; Granier, T.; Bousseksou, A.; Varret, F. Science 1995, 268, 265. (b) Roubeau, O.; Agricole, B.; Clerac, R.; Ravaine, S. J. Phys. Chem. B. 2004, 108, 15110. (c) Cobo, S.; Molnar, G.; Real, J. A.; Bousseksou, A. Angew. Chem., Int. Ed. 2006, 45, 5786. (d) Seredyuk, M.; Gaspar, A. B.; Ksenofontov, V.; Galyametdinov, Y.; Kusz, J.; Gütlich, P. J. Am. Chem. Soc. 2008, 130, 1431. (e) Cobo, S.; Ostrovskii, D.; Bonhommeau, S.; Vendier, L.; Molnar, G.; Salmon, L.; Tanaka, K.; Bousseksou, A. J. Am. Chem. Soc. 2008, 130, 9019.

(5) Coronado, E.; Galan-Mascaros, J. R.; Monrabal-Capilla, M.; GarciaMartinez, J.; Pardo-Ibanez, P. Adv. Mater. 2007, 19, 1359.

(6) (a) Bodenthin, Y.; Pietsch, U.; Mohwald, H.; Kurth, D. G. J. Am. Chem. Soc. 2005, 127, 3110. (b) Zhang, W.; Zhao, F.; Liu, T.; Yuan, M.; Wang, Z.-M.; Gao, S. Inorg. Chem. 2007, 46, 2541.

(7) (a) Tweedle, M. F.; Wilson, L. J. J. Am. Chem. Soc. 1976, 98, 4824. (b) Sinn, E.; Sim, G.; Dose, E. V.; Tweedle, M. F.; Wilson, L. J. J. Am. Chem. Soc. 1978, 100, 3375 .

(8) Solomon, E. I., Lever, A. B. P., Eds. Inorganic Electronic Structure and Spectroscopy; Wiley-Interscience: New York, 1999; Vol. 2.

(9) Evans, D. F. J. Chem. Soc. 1959, 2003.

(10) Complex $\mathbf{2 c}$, which is more soluble, undergoes a gradual spin transition over a broad temperature range, from $\mu_{\text {eff }}=5.44 \mu_{\mathrm{B}}$ at room temp to the low spin state $\left(\mu_{\mathrm{eff}}=2.47 \mu_{\mathrm{B}}\right)$ at $180 \mathrm{~K}$. A plot of $\mu_{\text {eff }}$ vs $T$ correlates with the increase of the absorbance at $650 \mathrm{~nm}$ and indicates that the spectroscopic changes indeed reflect a spin transition. 